\title{
Numerical Research of the Submerged High-Pressure Cavitation Water Jet Based on the RANS-LES Hybrid Model
}

\author{
Yongfei Yang $\left(\mathbb{D},{ }^{1}\right.$ Weidong Shi $\left(\mathbb{D},{ }^{1}\right.$ Linwei Tan $\left(\mathbb{D},{ }^{1}\right.$ Wei Li $\left(\mathbb{D},{ }^{2}\right.$ Songping Chen, ${ }^{2}$ and Bo Pan ${ }^{3}$ \\ ${ }^{1}$ College of Mechanical Engineering, Nantong University, Nantong 226019, China \\ ${ }^{2}$ Research Center of Fluid Machinery Engineering and Technology, Jiangsu University, Zhenjiang 212013, China \\ ${ }^{3}$ Shandong Xinchuan Mine Electrical and Mechanical Equipment Co., Ltd., Jining 272000, China \\ Correspondence should be addressed to Weidong Shi; wdshi@ujs.edu.cn and Linwei Tan; tanlinwei@ntu.edu.cn
}

Received 30 November 2020; Revised 21 April 2021; Accepted 15 June 2021; Published 12 July 2021

Academic Editor: Trupti Ranjan Lenka

Copyright ( $\odot 2021$ Yongfei Yang et al. This is an open access article distributed under the Creative Commons Attribution License, which permits unrestricted use, distribution, and reproduction in any medium, provided the original work is properly cited.

\begin{abstract}
The submerged high-pressure water jet has the characteristics of high velocity, strong turbulence, and severe cavitation. In order to reveal the formation mechanism of shear cavitation in the submerged high-pressure water jet and to grasp the turbulent structure and velocity distribution characteristics in the jet, the prediction ability of different turbulence models is studied first. The models represent the RANS model and RANS-LES hybrid model which are used to simulate the same cavitation jet, and the results are compared with the experimental results. The most reasonable model is then used to investigate the submerged high-pressure cavitation jet with different cavitation numbers. It is found that the calculation accuracy for small-scale vortexes has a great influence on the prediction accuracy of cavitation in the submerged jet. Both the DDES model and the SBES model can effectively capture the vortexes in the shear layer, and the SBES model can obtain more turbulence details. The result of the simulation under different cavitation numbers using the SBES model agrees well with the experimental result. Under the condition with low cavitation number, an intensive shear layer is formed at the exit of the nozzle, and small-scale vortexes are distributed along the shear layer. Mass transfer rate is relatively high in the region with a stronger vortex, which confirms that the low pressure in the vortex center is the main reason for the generation of cavitation in the shear layer. With the decrease of the cavitation number, the cavitation intensity increases obviously, while the nondimensional velocity along the radial direction changes little, which follows an exponential function.
\end{abstract}

\section{Introduction}

In recent years, with the implementation of energy-saving and environmental protection policies, the traditional cleaning technology for large-scale equipment with high pollution such as shot blasting is gradually eliminated. The high-pressure water jet cleaning technology has the characteristics of good processing quality, high efficiency, and environmentally friendly, which is gradually applied to the cleaning process of large-scale marine and chemical equipment [1].

The submerged high-pressure water jet is commonly accompanied by intensive cavitation; when the cavitation number is low and the bubble concentration is high, it is difficult to accurately measure the internal flow field by means of PIV or LDV. Therefore, it is of great significance to develop the numerical simulation technology of the submerged high-pressure water jet. After long-term research, the current CFD method has been able to accurately predict the velocity and pressure field of the submerged jets [2-9]. However, due to the complex phenomena involved in the cavitation jet, the model used in the calculation still needs to be further improved. At present, the main problems to be solved include mass transfer, turbulence, and compressibility. The mass transfer problem mainly corresponds to the modeling of cavitation dynamics. The turbulence problem is related to the accurate prediction of vortexes of various scales in the submerged jet, while compressibility will affect the periodic growth of cavitation unsteady characteristics such as shedding and collapse [10]. 
Currently, the most commonly used mass transfer models of cavitation include the Singhal model [11], Sauer model [12], and Zwart-Gerber-Belamri (ZGB) model [13]. The above models are based on the instantaneous pressure field to calculate the corresponding evaporation and condensation coefficients, which are converted into mass transfer rate and related to the mass and momentum transfer equations of each phase, so they are widely used in the multiphase flow model based on the Euler method. Hsiao et al. [14] modeled the cavitation process in another way. The Euler-Lagrange method was used to calculate the flow field of the cavitation flow. The position of the gas core in the flow field was tracked under the Lagrange coordinate. In this way, the limitation of homogeneous flow was avoided, and the multiscale distribution of cavitation bubbles was taken into consideration. At the same time, the aggregation and fragmentation of cavitation bubbles were considered in this kind of model. However, the Eulerian-Lagrangian method requires massive computational resources; as a result, it is not widely used at present. Cheng et al. [15] proposed a new Euler-Lagrange cavitation model based on the RayleighPlesset (R-P) equation, taking into account the noncondensable gas. In this model, the Euler method is used to solve the global flow field, and the Lagrangian method is used to track the migration of noncondensable gas bubbles into the vortex core. Based on the simplified R-P equation, the connection between local gas concentration and its effect on cavitation is modeled, and the mass source terms in the original Schnerr-Sauer (S-S) cavitation model are modified. $\mathrm{Xi}$ et al. [16] simulated the cavitating flow and pressure fluctuation in the tip region based on the delayed detached eddy simulation (DDES). The high-speed photography and transient pressure measurements were employed to capture the cavitation structures and pressure fluctuation. The numerical results showed a reasonable agreement with the available experiments.

In the research of submerged jet turbulence, different turbulence models were used to simulate free jet and impinging jet with different Reynolds numbers. Yang et al. [17] employed the SST $k$ - $\omega$ turbulence model to capture the nearwall characteristics of the flow field within the impellers and diffusers, which has the advantage to predict the flow separation under adverse pressure gradients. Yang et al. [18] used ANSYS CFX 17.0 to do the numerical calculations. The SST $k$ - $\omega$ turbulence model is selected, which is capable of solving turbulence parameters very close to the blades. Miltner et al. [19] carried out numerical simulation and experimental research on the submerged swirling jets. Various RANS models were used to calculate the flow field to obtain the velocity distribution at different positions of the jet. At the same time, LDV technology was used to measure the flow field. By comparing the velocity and turbulent kinetic energy distribution obtained by the simulation and experiment, it was found that the standard $k-\varepsilon$ model has a better prediction for the detailed characteristics of various regions of the jet. Saddington et al. [20] studied the supersonic jet of an axisymmetric contraction nozzle, found that the calculation results of the RNG $k-\varepsilon$ model were in good agreement with the experimental results, and accurately predicted the position and intensity of the shock wave generated by the supersonic jet. Jaworski and Zakrzewska [21] used the inclined blade impeller to form the impinging jet to the bottom in the agitator and carried out the numerical calculation and experimental research on the impinging jet at the same time. It was also found that the $k-\varepsilon$ model was the most accurate for the calculation of the averaged velocity and turbulent kinetic energy of the flow field. Birkby and Page [22] used the $k-\varepsilon$ model to simulate the underexpanded supersonic jet. In the simulation process, the pressure-based solver was used to iterate, and the compression performance was modified to accurately predict the position of the Mach disk. Wang et al. [23] used large eddy simulation to simulate the heat transfer process of the impinging jet, accurately obtained the heat transfer process of the jet cooling device through numerical calculation, and proposed a design method of the jet cooling device based on numerical simulation. Tip leakage vortex (TLV) flow is a common flow phenomenon in the axial-flow hydraulic machinery. High-efficiency simulation of TLV is still not an easy task because of the complex turbulent vortex-cavitation interactions. Wang et al. [24] evaluated the newly developed MST turbulence model in predicting the TLV flows.

However, in the case of high-pressure submerged jet, especially when intensive cavitation occurs, the turbulence model has a great effect on the prediction accuracy of the mass transfer phenomenon, while the research on the relation between the turbulence model and the cavitation formation process in the high-pressure submerged jet is lacking. The high computational cost of the LES model hinders the promotion of large eddy simulation technology in practical applications, so there is no discussion. The RANS model can no longer meet the accuracy requirements in some research and application fields, the RANSLES hybrid model is to solve the boundary layer with the RANS model, and the separated flow is solved through the LES models. In this way, the accuracy of the flow field solution is improved while avoiding the excessive number of grids caused by the boundary layer analysis. In this paper, three different turbulence models that represent the RANS and RANS-LES hybrid model are used, and the probability for the models to simulate the microscale vortex structures is compared. By analyzing the relation between the small-scale vortex and the cavitation formation, the probability for these models to simulate the high-pressure cavitation jet with strong shear stress is discussed. The mechanism and the characteristics of the vortex and cavitation cloud evolution process of the submerged highpressure jet are revealed.

\section{Theoretical Models}

2.1. Multiphase Model. Cavitation jet belongs to gas-liquid two-phase flow. In order to reduce the amount of calculation, the Euler model is usually used for the calculation of gas-liquid two-phase flow when the individual motion characteristics of the discrete phase are not concerned. In this paper, the mixture model is used to calculate the mixedphase flow field, and the control equation is as follows [25]: 


$$
\begin{aligned}
\frac{\partial}{\partial t}\left(\rho_{m}\right)+\nabla \cdot\left(\rho_{m} \vec{v}_{m}\right)= & \\
\frac{\partial}{\partial t}\left(\rho_{m} \vec{v}_{m}\right)+\nabla \cdot\left(\rho_{m} \vec{v}_{m} \vec{v}_{m}\right)= & -\nabla p+\nabla \cdot\left[\mu_{m}\left(\nabla \vec{v}_{m}+\vec{v}_{m}^{T}\right)\right] \\
& +\rho_{m} \vec{g}+\vec{F}+\nabla \cdot\left(\sum_{k=1}^{n} \alpha_{k} \rho_{k} \vec{v}_{d r, k} \vec{v}_{d r, k}\right)
\end{aligned}
$$

where $\vec{v}_{m}$ is the mass-averaged velocity, $\rho_{m}$ is the density of the mixture,

$$
\rho_{m}=\sum_{k=1}^{n} \alpha_{k} \rho_{k},
$$

and $\mu_{m}$ is the viscosity of the mixture, which is defined as follows:

$$
\mu_{m}=\sum_{k=1}^{n} \alpha_{k} \mu_{k}
$$

where $n$ is the number of phases, $\vec{F}$ is the volume force, and $\vec{v}_{d r, k}$ represents the slipping velocity of phase $k$.

2.2. Cavitation Model. When the mixture model is used to calculate the cavitation multiphase flow, the expression of the gas volume fraction transport equation is as follows [26]:

$$
\frac{\partial}{\partial t}\left(\alpha \rho_{v}\right)+\nabla \cdot\left(\alpha \rho_{v} \vec{v}_{v}\right)=R_{e}-R_{c}
$$

where $R_{e}$ and $R_{c}$ are evaporation and condensation rates, and their values can be calculated according to the cavitation model.

At present, the commonly used cavitation models are mainly derived from the Rayleigh-Plesset equation. The cavitation model used in this paper to calculate the phase transition is the Zwart-Gerber-Belamri model, and the mass transfer expression of the model is as follows:

$$
\begin{aligned}
& R_{e}=F_{\mathrm{vap}} \frac{3 \alpha_{\mathrm{nuc}}\left(1-\alpha_{v}\right) \rho_{v}}{R_{B}} \sqrt{\frac{2}{3} \frac{P_{v}-P}{\rho_{l}}}, \\
& R_{c}=F_{\text {cond }} \frac{3 \alpha_{v} \rho_{v}}{R_{B}} \sqrt{\frac{2}{3} \frac{P-P_{v}}{\rho_{l}}}
\end{aligned}
$$

where $R_{B}$ is the radius of the cavity, $\alpha_{\text {nuc }}$ is the volume fraction of the gas core in the liquid, $F_{\text {vap }}$ is the evaporation coefficient, and $F_{\text {cond }}$ is the condensation coefficient. Considering the influence of turbulence on cavitation, the expression of the pressure threshold for cavitation occurrence is as follows:

$$
P_{v}=P_{\text {sat }}+\frac{1}{2} c \rho_{1} k_{1},
$$

where $\rho_{1}$ and $k_{1}$ represent the liquid phase density and liquid phase turbulent kinetic energy, respectively, and the coefficient $c$ adopts the default value of 0.39 .

\subsection{Turbulence Model}

2.3.1. RANS Models. Although the scale of the cavitation jet nozzle is relatively small, the nozzle outlet velocity is extremely high, so the Reynolds number of the submerged jet is large. The reasonable choice of the turbulence model has a great influence on the accuracy of the calculation results of the cavitation jet. This paper uses Reynolds time average (RANS) and hybrid (RANS-LES) models to perform numerical calculations on submerged cavitation jets. The calculation results are compared with experimental results, and the analysis is processed. The turbulence flow structure in the shear layer is compared for different turbulence models, and the applicability of the RANS model to calculate the cavitation jet is evaluated. In the RANS model, SST $k-\omega$ has a good calculation effect on the vortex structure, so this paper will choose this model as the representative of the RANS model to simulate the cavitation jet, whose expression is as follows [27]:

$$
\begin{aligned}
\frac{\partial}{\partial t}(\rho k)+\frac{\partial}{\partial x_{i}}\left(\rho k u_{i}\right) & =\frac{\partial}{\partial_{x_{j}}}\left(\Gamma_{k} \frac{\partial k}{\partial x_{j}}\right)+G_{k}-Y_{k}+S_{k}, \\
\frac{\partial}{\partial t}(\rho \omega)+\frac{\partial}{\partial x_{i}}\left(\rho \omega u_{i}\right) & =\frac{\partial}{\partial x_{j}}\left(\Gamma_{\omega} \frac{\partial \omega}{\partial x_{j}}\right)+G_{\omega}-Y_{\omega}+D_{\omega}+S_{\omega}, \\
\Gamma_{k} & =\mu+\frac{\mu_{t}}{\sigma_{k}}, \\
\Gamma_{\omega} & =\mu+\frac{\mu_{t}}{\sigma_{\omega}},
\end{aligned}
$$

where $G_{k}$ is the generation term of turbulent kinetic energy, $G_{\omega}$ is the generation term of dissipation rate $\omega, Y_{k}$ and $Y_{\omega}$ represent the dissipation of $k$ and $\omega$ caused by turbulence, $\Gamma_{k}$ and $\Gamma_{\omega}$ are the effective diffusivity of $k$ and $\omega$, and $\sigma_{k}$ and $\sigma_{\omega}$ are the turbulence Prandtl number of $k$ and $\omega$, respectively. $\mu_{t}$ represents the turbulent viscosity, which is calculated by the following formula:

$$
\begin{aligned}
\mu_{t} & =\frac{\rho k}{\omega} \frac{1}{\max \left[\left(1 / \alpha^{*}\right),\left(S F_{2} / a_{1} \omega\right)\right]}, \\
F_{2} & =\tanh \left(\Phi_{2}^{2}\right), \\
\Phi_{2} & =\max \left[2 \frac{\sqrt{k}}{0.09 \omega y}, \frac{500 \mu}{\rho y^{2} \omega}\right] .
\end{aligned}
$$


2.3.2. RANS-LES Hybrid Models. With the continuous development of CFD technology, the RANS model can no longer meet the accuracy requirements in some research and application fields, and the high computational cost of the LES model hinders the promotion of large eddy simulation technology in practical applications. In order to solve the above problems, the RANS-LES hybrid model has gradually gained more attention in recent years. Among them, the separation vortex (DES) model is currently the most widely used hybrid model. This model was first proposed by Spalart [28] in 1997, so the original version of the DES model is also called DES97. The main idea of the DES model is to solve the boundary layer with the RANS model, and the separated flow is solved through the LES models. In this way, the accuracy of the flow field solution is improved while avoiding the excessive number of grids caused by the boundary layer analysis. To avoid the problem of the gridinduced separation (GIS), the boundary layer is further treated, and the model is called delayed detached eddy simulation (DDES).

(1) Detached Eddy Simulation. The expression of the turbulence energy dissipation term of the DDES model used in this paper is as follows:

$$
\begin{aligned}
Y_{\mathrm{k}} & =\rho \beta^{*} k \omega F_{\mathrm{DES}}, \\
F_{\mathrm{DES}} & =\max \left(\frac{L_{t}}{C_{\mathrm{des}} \Delta_{\max }}\left(1-F_{\mathrm{DDES}}\right), 1\right), \\
F_{\mathrm{DDES}} & =\tanh \left[\left(C_{d 1} r_{d}\right)^{C_{d 2}}\right], \\
r_{d} & =\frac{v_{t}+v}{k^{2} y^{2} \sqrt{0.5\left(S^{2}+\Omega^{2}\right)}},
\end{aligned}
$$

where $C_{d 1}=20, C_{d 2}=3, S$ is the strain tensor size, $\Omega$ is the vorticity tensor, and $k=0.41$.

(2) Stress-Blended Eddy Simulation. In the process of studying the mixed model, a stress-mixed vortex model (SBES) appeared after the DES model. The shielding function used in this type of model is the same as the DES form. The SBES model can directly realize RANS and stress mixing between LES:

$$
\tau_{i j}^{\mathrm{SBES}}=f_{\mathrm{SDES}} \tau_{i j}^{\mathrm{RANS}}+\left(1-f_{\mathrm{SDES}}\right) \tau_{i j}^{\mathrm{LES}}
$$

where $\tau_{i j}^{\mathrm{RANS}}$ and $\tau_{i j}^{\mathrm{LES}}$ represent the stress tensor of RANS and LES parts.

\section{Mesh and Boundary Conditions}

Cavitation jet nozzles usually adopt special structures to improve the cavitation performance. Commonly used structures include convergent-divergent nozzles, organ tube nozzles, Helmholtz nozzles, and center body nozzles. Considering practicability and ease of processing, the first two nozzles are more widely used in high-pressure water jet cleaning, oil extraction, and peening. The current research mainly focuses on a convergent-divergent nozzle, and the nozzle structure is shown in Figure 1. The specific

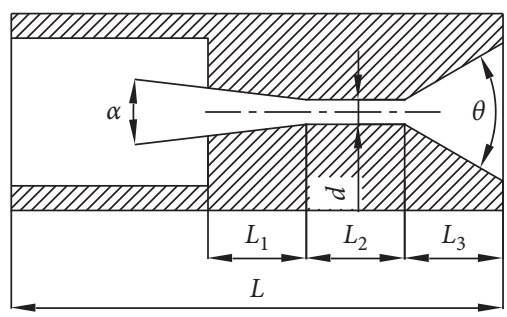

FIGURE 1: Geometry structure of the nozzles used in the experiment and simulation.

parameters are as follows: the convergent angle $\alpha=13.5^{\circ}$, length of the convergent section $L_{1}=5 \mathrm{~mm}$, throat length $L_{2}=4 \mathrm{~mm}$, throat diameter $d=1 \mathrm{~mm}$, outlet section length $L_{3}=4 \mathrm{~mm}$, and outlet expansion angle $\theta=40^{\circ}$.

Figure 2 shows the calculation domain of the submerged jet. The incoming flow should develop fully in the pipe before reaching the nozzle, so the pipe at the nozzle inlet is extended by $260 \mathrm{~mm}$ upstream. In order to reduce the influence of entrainment and outlet reversed flow on the core area of the jet, the calculation boundary should be as far away as possible from the nozzle outlet. According to the literature, the diameter of the calculation domain should be greater than 100 times the diameter of the nozzle throat. In this paper, the diameter of the submerged water calculation domain is $200 \mathrm{~mm}$. A cylinder is with a length of $500 \mathrm{~mm}$, and the nozzle diameter used in the current calculation is $1 \mathrm{~mm}$. The calculation domain size meets the above requirements.

Yun et al. [29] found that when the mesh resolution improves, the predicted velocity distributions and the cavitation patterns are in better agreement with the experimental results. Moreover, only when the mesh resolution reaches a certain high degree can the predicted cavitation results reflect the effects of the vortex stretching in the unsteady cloud cavitation. The computational domain is divided into structured grids using ANSYS ICEM, and the core area of the jet and the shear layer near the nozzle exit are refined. The computational domain grid is shown in Figure 3. In order to minimize the requirement for the calculation resource, the mesh size is gradually increased from the nozzle outlet to the downstream.

GIS has been a problem for DES models since the DES97 model; in the current research, different meshes are used to test the effect of the grid on the simulation results. The GIS characteristics of both DES and SBES models are investigated by refining the mesh gradually in the region of the throat and the outlet section. The total grid numbers are shown in Table 1.

Figure 4 shows the skin friction coefficient on the surface of the nozzle divergent section. It can be seen that the skin friction coefficient varies with the change of the grid number, while the result by SBES and DDES has few differences when the mesh is not very fine. Since the DDES model has a delayed mechanism to protect the boundary from GIS, the turbulence viscosity in the boundary layer is kept in the range for the RANS simulation. The result reveals that the current used SBES model also treats the boundary 


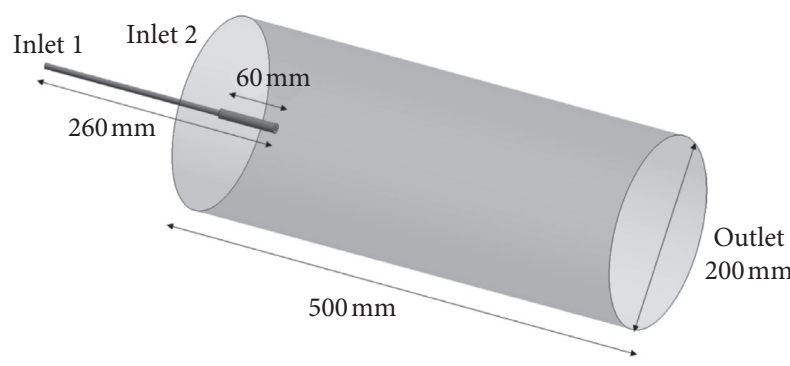

Figure 2: Computational domain of the submerged jet.

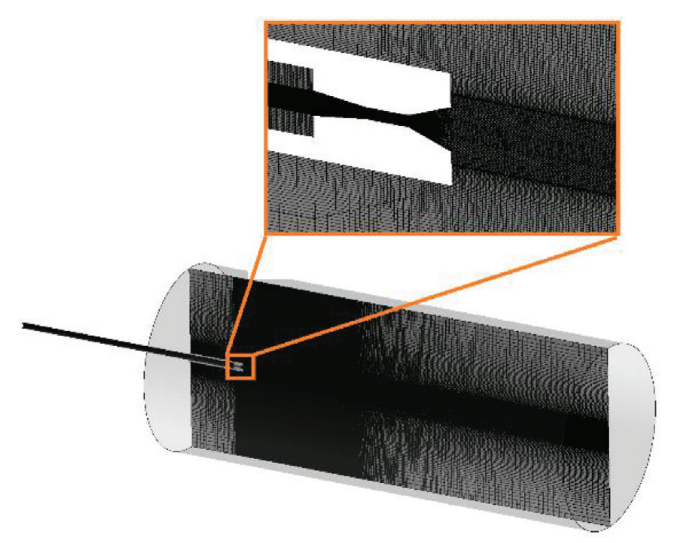

Figure 3: Mesh of the submerged jet domain.

TABLe 1: Node number of the mesh used for GIS analysis.

\begin{tabular}{lc}
\hline Mesh & Node number \\
\hline M1 & $2,501,982$ \\
M2 & $7,940,816$ \\
M3 & $19,505,920$ \\
\hline
\end{tabular}

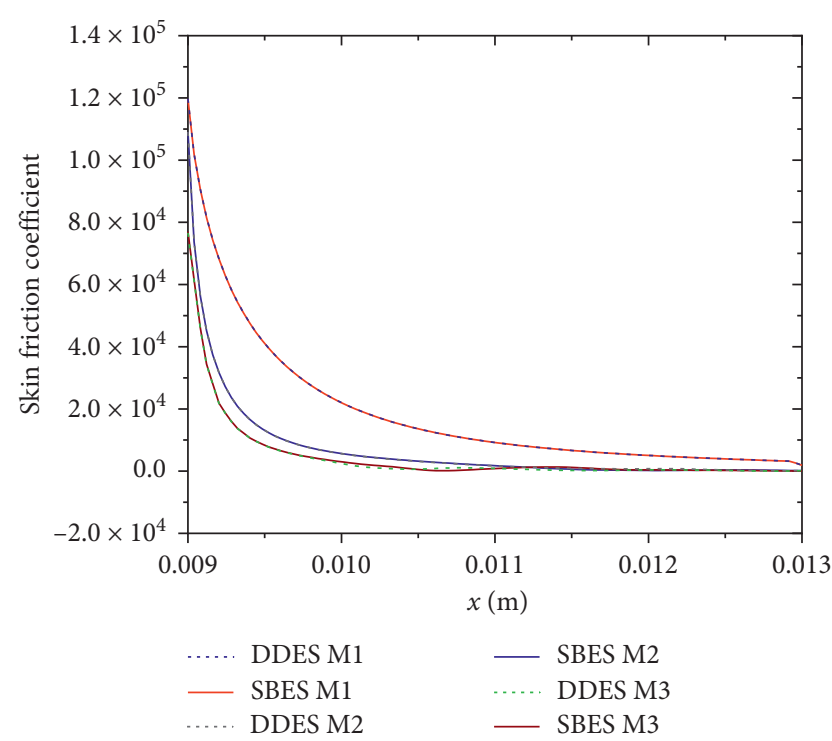

Figure 4: Skin friction coefficient on the surface of the nozzle divergent section. layer well in a RANS way. When the mesh is refined and the cell number is larger than 13 million, the skin friction coefficient of SBES differs from that of DDES. The result shows that the boundary layer of the DDES model tends to separate. With the refinement of the mesh, the variation of skin friction coefficient at the nozzle outlet becomes less, which remains almost unchanged when the mesh is larger than 19 million. Finally, the mesh with a cell number of around 19 million was used for the later simulation research studies.

According to the water domain structure shown in Figure 3, combined with the experimental conditions, the calculation boundary conditions are set. The calculation domain is meshed based on the finite volume method, and the pressure-velocity coupling method is used to solve the problem. The volume fraction transport equation uses the first-order upwind difference, the transient term uses the second-order central difference, the continuity equation, the momentum equation, and the turbulence control equation are discretized using the second-order upwind difference scheme. The inlet of the high-pressure pipe is set according to the corresponding cavitation number. The reference pressure in the calculation is $101,325 \mathrm{~Pa}$. The outlet is set as the pressure outlet, the gauge pressure value is $0 \mathrm{MPa}$, and the other boundaries are set to nonslip wall. Since the cavitation jet is an unsteady phenomenon, transient calculations are used in all calculation examples. Due to the high velocity at the nozzle exit, in order to control the Courant number in the calculation, the calculation time step should be reduced as much as possible, which is set as $0.00001 \mathrm{~s}$. The calculation accuracy is $1 \times 10^{-6}$, and the maximum number of iterations in each time step is 20 times. The total number of steps is 2000 steps.

\section{Results and Discussion}

4.1. Effect of Turbulence Models and Mesh. The choice of the turbulence model determines the accuracy of the velocity field, pressure field, velocity and pressure pulsation, and other physical quantities. Therefore, it is necessary to select a suitable turbulence model before conducting numerical simulation studies of cavitation jets. This paper uses the time-average model (RANS) and two hybrid models (RANSLES) to simulate high-pressure water cavitation jets. The simulated boundary conditions are set according to the experimental conditions. The inlet pressure of the highpressure pipeline is set to $20 \mathrm{MPa}$, and the corresponding cavitation number is $\sigma=0.005$.

Figure 5 shows the instantaneous velocity distribution of submerged jets calculated by three different turbulence models. The RANS model used is the SST $k$ - $\omega$ model, and the hybrid model uses DDES and SBES based on the SST $k-\omega$ model. According to the calculated velocity field, it can be seen that the jet velocity field predicted by the above models is basically reasonable. The velocity in the core area near the nozzle exit is the highest, the velocity gradually decays along the axis, and the velocity decays to zero when it reaches $150 \mathrm{~mm}$ downstream of the nozzle. At the same time, under the action of the viscous force of the shear layer, the highspeed jet entrains the surrounding fluid, and the process of 

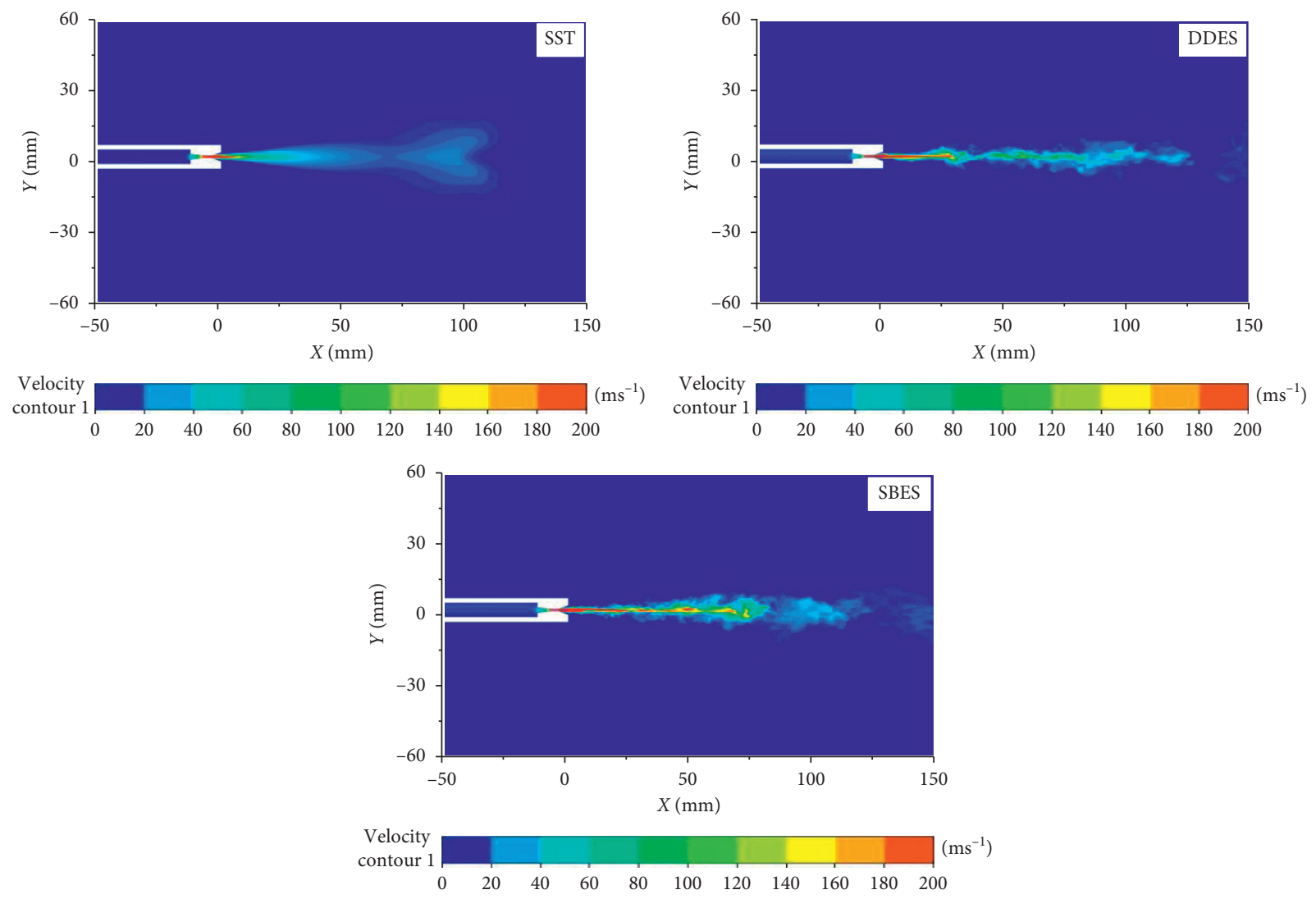

FIGURE 5: Transient velocity calculated using different turbulence models.

velocity attenuation from the nozzle to the downstream is accompanied by radial diffusion. The SST $k-\omega$ model has a smooth transition from the core area to the periphery due to the time-averaged effect, which can basically reflect the spatial distribution of the submerged jet velocity under this working condition. In the results calculated by the DDES models, the high-speed core area extends from the nozzle to about $30 \mathrm{~mm}$ downstream. At the same time, when the velocity field decays, there is no smooth contour but a fuzzy gradient boundary, which is consistent with the dissipation of large-scale vortexes to small-scale vortexes under the action of viscous forces in the turbulent flow field. Compared with the DDES model, the velocity field calculated by the SBES model can reflect the small-scale vortex structure at the edge of the shear layer from the nozzle exit, and the core high-speed zone is intermittently distributed along the axis. The intermittent distribution indicates that the model can better reflect the unsteady velocity pulsation of the cavitation jet flow field.

Figure 6 shows the calculated void volume fraction distribution of the submerged jet. It can be seen that the shape of the void cloud obtained by the last two turbulence models is relatively similar on the whole and is close to the real cavitation form of the high-pressure submerged jet, while the cavitation calculated by the SST $k-\omega$ model is only distributed in the $10 \mathrm{~mm}$ long interval of the nozzle outlet, which is quite different from the real situation observed in the experiment, indicating that the SST $k$ - $\omega$ model is affected by the time-average processing and cannot accurately capture the shear vortex structure and the low-pressure vortex core caused by it. Comparing the three turbulence models, it can be seen that the cavitation range and concentration in the shear layer near the nozzle outlet increase sequentially. The cavitation cloud calculated by the DES model is a discontinuous cluster distribution, and the cavitation cloud calculated by the SBES model is distributed continuously.

At present, the mainstream explanation for the mechanism of submerged jet cavitation is that a Rankine combined vortex is formed between the high-speed jet and the static submerged water under the action of shear. The vortex of this structure is composed of two parts inside and outside. The velocity and pressure value are lower near the center, and the cavitation occurs when the central pressure of the Rankine vortex is lower than the saturated vapor pressure of the liquid. Therefore, whether the cavitation process of the high-pressure water submerged jet can be accurately predicted has put forward higher requirements for the accuracy of the vortex structure prediction. Figure 7 shows the vorticity distribution in the jet flow field calculated by the compared models. It can be seen that the vorticity distribution is similar to the distribution of the cavitation cloud. This is because the vortex and turbulence affect the cavitation in the flow field. The vorticity calculated by the SST $k$ $\omega$ model is mainly concentrated in the nozzle outlet section. The area with a larger vorticity value is basically the same as 


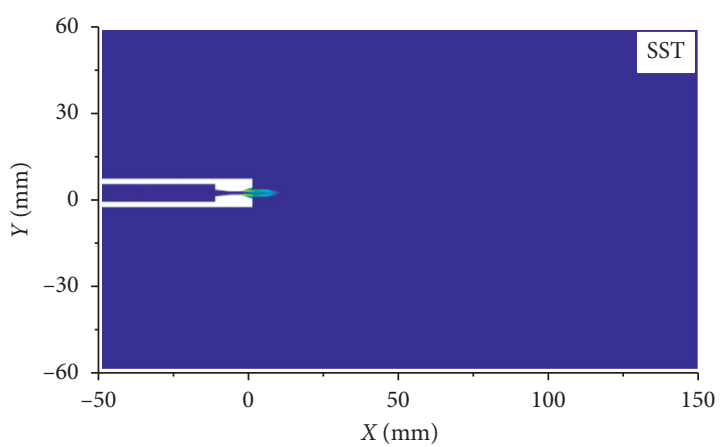

Phase 2

volume

fraction

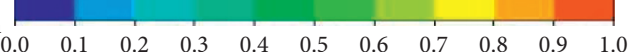

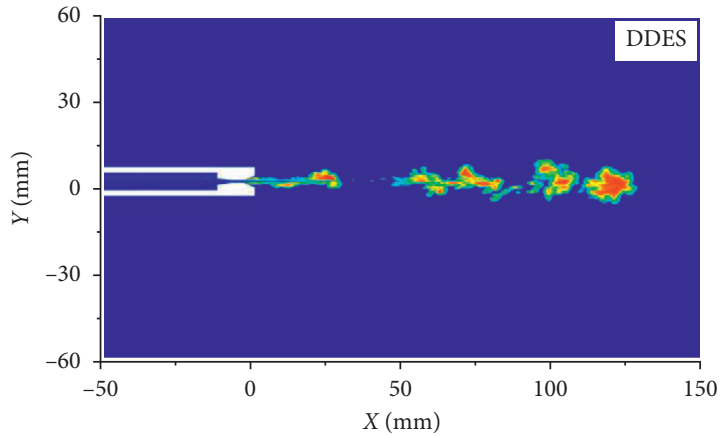

Phase 2

volume

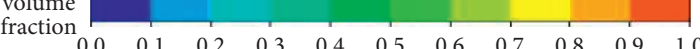

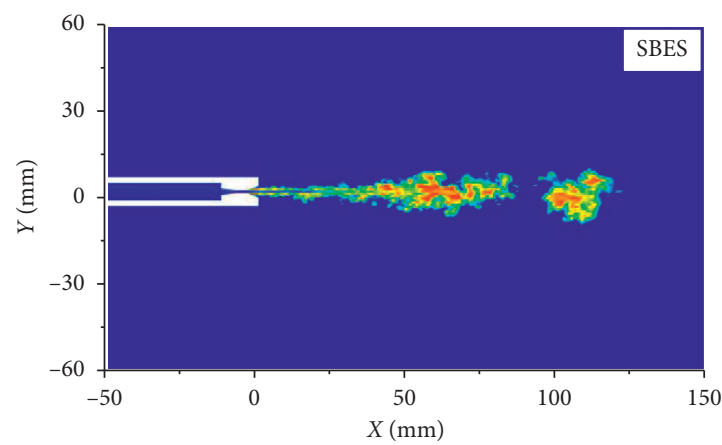

Phase 2

volume

$\begin{array}{rllllllllll}\text { fraction }_{0.0} & 0.1 & 0.2 & 0.3 & 0.4 & 0.5 & 0.6 & 0.7 & 0.8 & 0.9 & 1.0\end{array}$

FIGURE 6: Vapor volume fractions by different turbulence models.
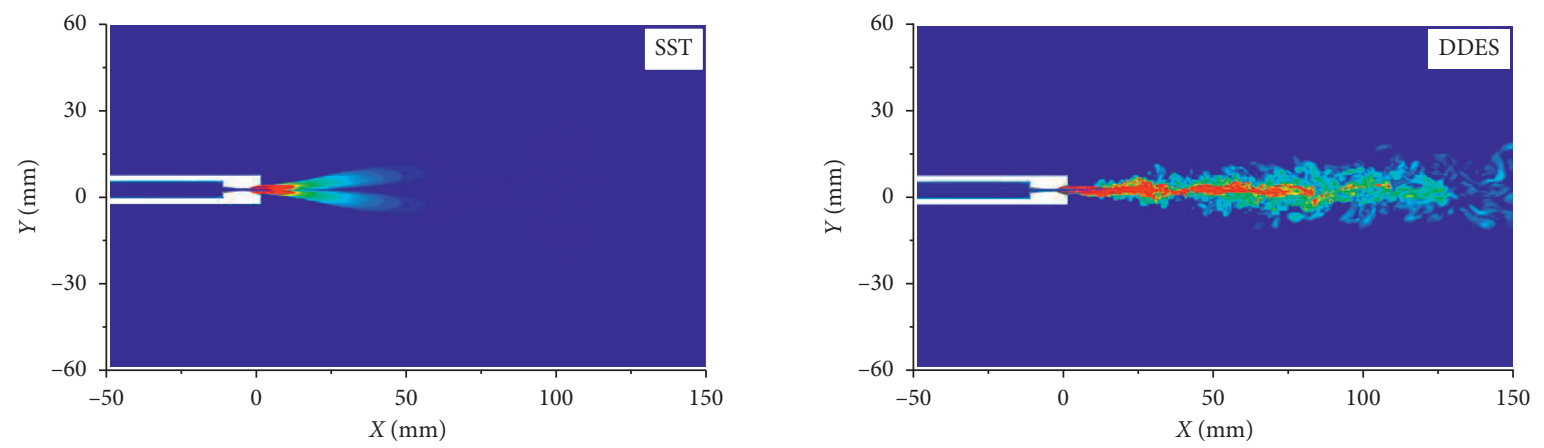

Vorticity

Vorticity

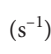

$0 \quad 5000100001500020000250003000035000400004500050000$

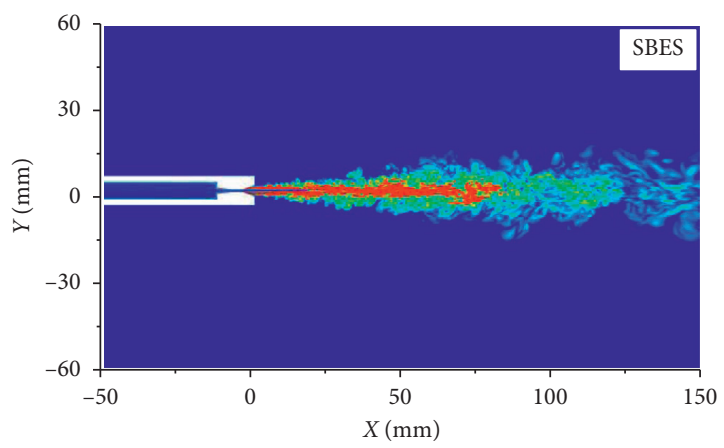

Vorticity

\section{$\left(\mathrm{s}^{-1}\right)$}

$0 \quad 5000100001500020000250003000035000400004500050000$

Figure 7: Vorticity of the jet by different turbulence models. 
the cavitation area, and the transition of the vorticity isosurface is smooth. The vorticity calculated by the DES models clearly reflects the characteristics of turbulence. The vorticity boundary of the nozzle outlet section is relatively smooth, and the transition begins at about $10 \mathrm{~mm}$ downstream. A vortex appears around the shear layer, and the vortex scale increases gradually along the mainstream direction. The results of the SBES model are similar to those of the DES model, and the difference is that the position where the vortex begins to appear is closer to the nozzle.

Through calculation and comparison, it is found that the SST $k$ - $\omega$ model is affected by the time-average processing when simulating the submerged high-pressure water cavitation jet, and the corresponding scale of turbulence and vortex structure cannot be obtained. Therefore, the calculated local pressures are average values, which affect the capture of cavitation phenomena. The two hybrid models are ideal for the calculation results of high-pressure submerged jets. They can capture the vortexes generated by the viscous force of the shear layer, as well as the merger and dissipation of the vortexes. The shape, size, and development period of the cavitation cloud are reasonable compared to the experiment. Comparing the two hybrid models, the SBES model has a better probability to capture details in the shear layer.

Besides the GIS phenomenon, mesh size is found to have a great effect on the vapor distribution in shear cavitation flows. Figure 8 shows the isosurface of 0.5 vapor volume fraction with different turbulence models and mesh sizes. It can be found that the shape of the cavity changes with the refinement of the mesh, and more small vortex structures can be seen in the case of the fine meshes. Generally, the result of the SBES model improves more by refining the mesh. For the hybrid models, the region where the vortex is solved is decided by the shielding function, which defines the region where the turbulence viscosity is modeled. Figure 9 shows the isosurface of the vorticity colored by eddy viscosity ratio. It can be found that the eddy viscosity ratio is decreased when the mesh is refined. Comparing the result of the same mesh with different models, one can find that the result of the SBES model shows lower eddy viscosity, and the jet transfers faster to turbulent under the effect of the shear stress when the jet flows from the nozzle outlet to downstream.

4.2. Validation of the Simulation Result. Before analyzing the high-pressure water submerged cavitation jet using the current simulation model, a nozzle with a $40^{\circ}$ expansion angle is taken for validation. The numerical calculation method and its accuracy are verified by comparing the simulation results with the high-speed photography results. Figure 10 shows the comparison of the cavitation morphology obtained by high-speed photography and numerical simulation of the selected nozzle with different turbulence models. It can be found from the figures that the shape of the cavitation cloud predicted by the SST $k-\omega$ model is obviously different from the reality. The two kinds of hybrid models can provide good results, which are similar to the high-speed photograph result both in shape and scale. Observing the high-speed image, small-scale vortex structures can be seen from the region close to the nozzle outlet. Comparing the results by the two types of hybrid models, it can be seen that SBES provides more detailed shear vortexes near the nozzle outlet. As a result, the later numerical investigation on the high-pressure cavitation jet is conducted using the SBES model.

4.3. Vortex and Cavitation Characteristics. High-pressure water submerged jets usually have a relatively high central velocity. When the high-speed liquid rushes into still submerged water, there is a large velocity gradient at the boundary layer. The boundary layer is filled with shear stress and reverses pressure difference. For small-scale vortexes, because the pressure at the center of the vortex is low, cavitation will occur when the pressure at the center of the vortex is lower than the saturated vapor pressure at the ambient temperature where the liquid is located. When the jet keeps shearing submerged water at a high speed, the shear layer will always be full of Rankine vortexes, and cavitation in the low-pressure zone will occur continuously, and the bubbles will travel downstream with the mainstream. Figure 11 shows the velocity vector diagram at the exit of the angular nozzle. It can be clearly seen from the figure that the velocity in the core area is as high as $200 \mathrm{~m} / \mathrm{s}$, while submerged water at the periphery is almost stationary. Shearing layer thickness at the exit of the throat is on the order of micrometers, which indicates that there must be a strong velocity gradient in the shear layer. Under the action of viscous force and reverse pressure difference, the phenomenon of $\mathrm{K}-\mathrm{H}$ instability appears. The shear layer is rapidly destabilized from the ideal shear layer, and the fluids on both sides begin to exchange momentum to form a vortex structure. The process of vortexes propagating downstream is accompanied by a pairing process. Small-scale vortexes in the shear layer continue to increase through merging, so the thickness of the shear layer continues to increase, and the jet velocity field also diffuses.

Figure 12 shows the distribution of various physical quantities near the shear layer of the nozzle outlet section. From the cavitation vapor volume fraction in Figure 12(a), it can be seen that the cavitation of the nozzle starts from the junction between the throat and whistle. The cavitation bubbles are mainly distributed along the boundary of the shear layer near the exit of the nozzle. Affected by pressure fluctuations, cavitation near the shear layer is also accompanied by the collapse of a large number of bubbles. The shock wave generated during the collapse aggravated the collapse of the surrounding cavitation. The alternate generation and collapse of the cavitation formed the development process of the cyclical growth, shedding, and collapse of the cavitation cloud as captured by the high-speed photography experiment. Figure 12(b) shows the mass transfer rate between vapor and liquid phases. A positive value represents the occurrence of cavitation, while a negative value represents the collapse of cavitation bubbles. It can be seen that, except for the exit of the throat, the area 


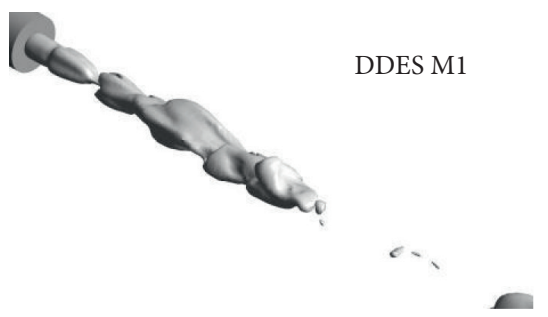

(a)

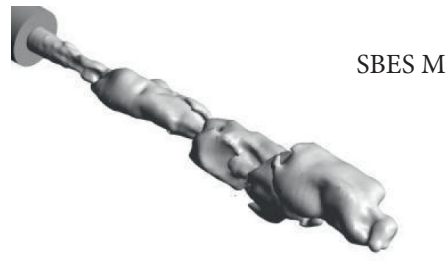

(d)

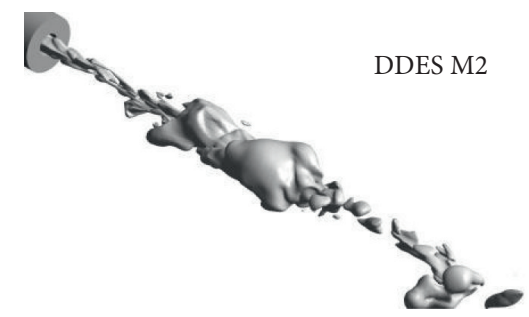

(b)

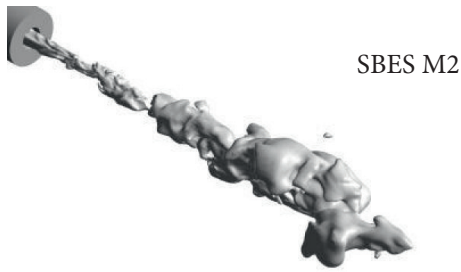

(e)

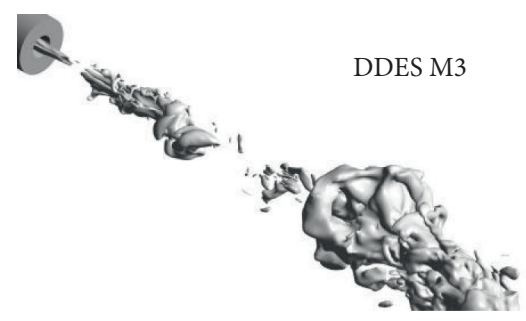

(c)

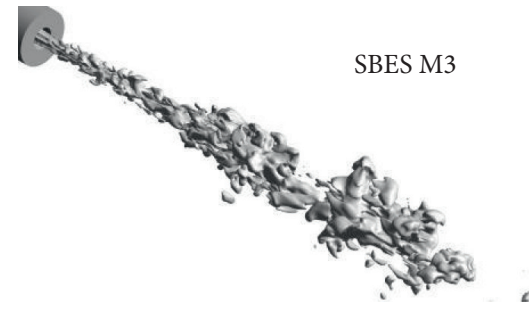

(f)

Figure 8: Shape of vapor with different meshes and turbulence models.

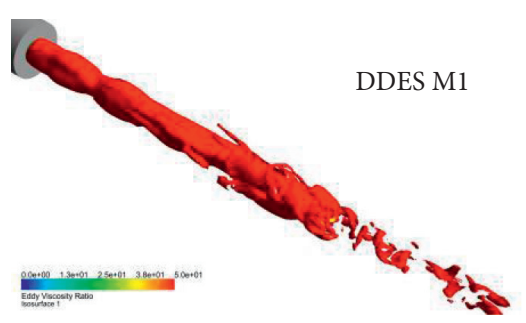

(a)

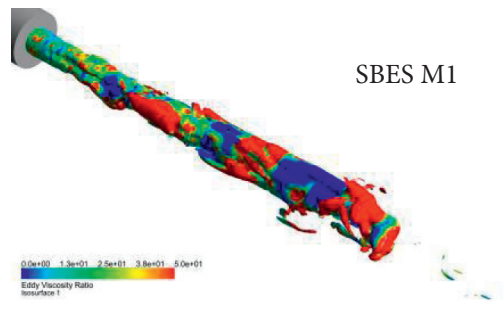

(d)

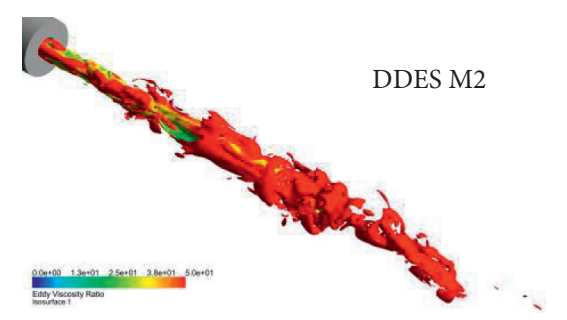

(b)

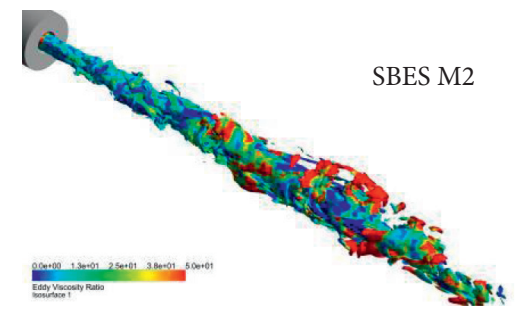

(e)

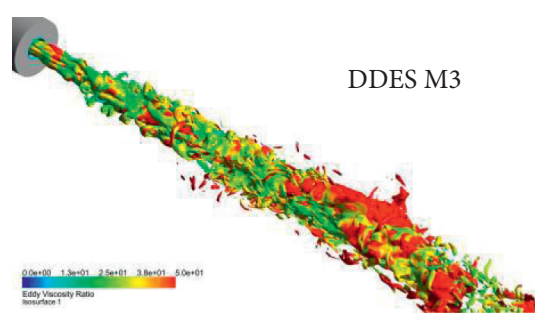

(c)

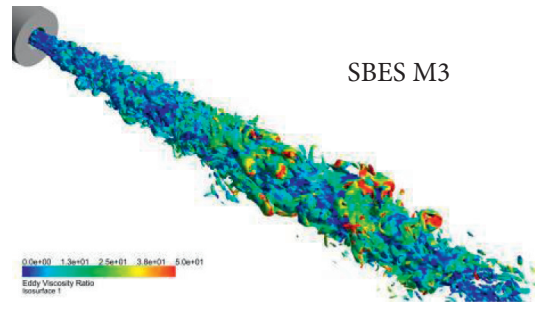

(f)

FIGURE 9: Isosurface of vorticity colored by eddy viscosity ratio.

where cavitation occurs is basically circular in cross section, which corresponds to the vortex ring formed by shear in the space. Figure 12(c) shows the distribution of the vortex criterion $Q$ value, which is defined as follows:

$$
Q=\frac{1}{2}\left(\|\Omega\|^{2}-\|\mathrm{E}\|^{2}\right)=\frac{1}{2}\left(\Omega_{i j} \Omega_{i j}-e_{i j} e_{j i}\right),
$$

where $\Omega_{i j}$ and $e_{i j}$ represent the vorticity tensor and strain rate tensor, respectively; when $\|\Omega\|^{2}-\|\mathrm{E}\|^{2}>0$, it means that the effect of the local fluid rotation overwhelms the strain.

It can be seen that the $Q$ value in the shear layer alternates between positive and negative. Under the effect of the shear stress, the viscous fluid must have a strain rate, and the positive $Q$ value represents the formation of vortexes. The characteristics of the vortexes are gradually increased streamwise. On the one hand, decrease of the core velocity in the downstream leads to a decrease in shear stress. On the other hand, the scale of the vortexes gradually increases during the vortex pairing process. Figure 12(d) shows the distribution of turbulent kinetic energy near the shear layer. The area with higher turbulent kinetic energy corresponds to a stronger vortex. Comparing the volume fraction of bubbles, it can be found that the turbulent kinetic energy is the highest at the gas-liquid interface, indicating that, in such areas, the flow field disturbance is increased due to the momentum exchange between the two phases.

In industrial applications, jets of different pressures and flows correspond to different application conditions. When nozzles, pipelines, and other systems are determined, it is generally necessary to select a reasonable operating pressure according to the requirements. When the pressure upstream of 


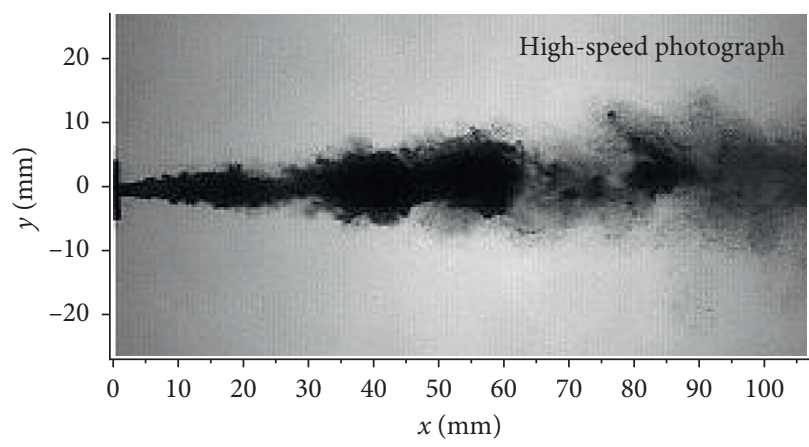

(a)

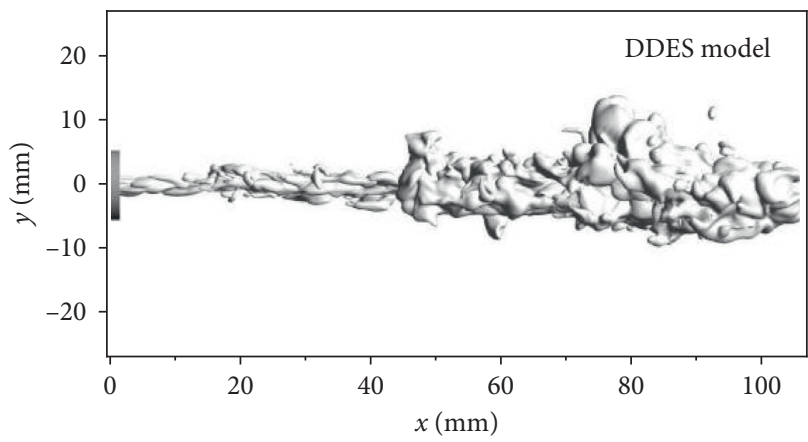

(c)

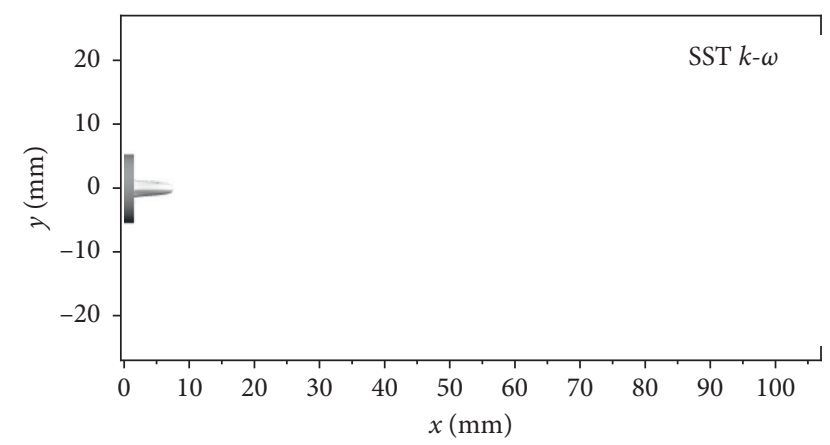

(b)

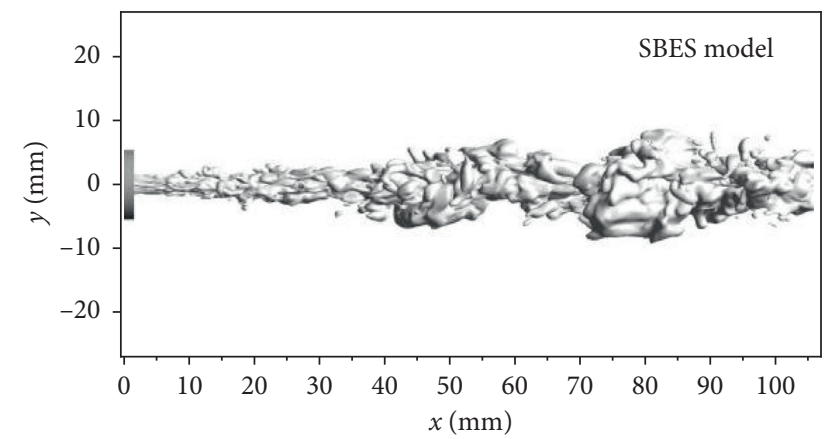

(d)

FIGURE 10: Morphology of the cavitation clouds by experiment and CFD.
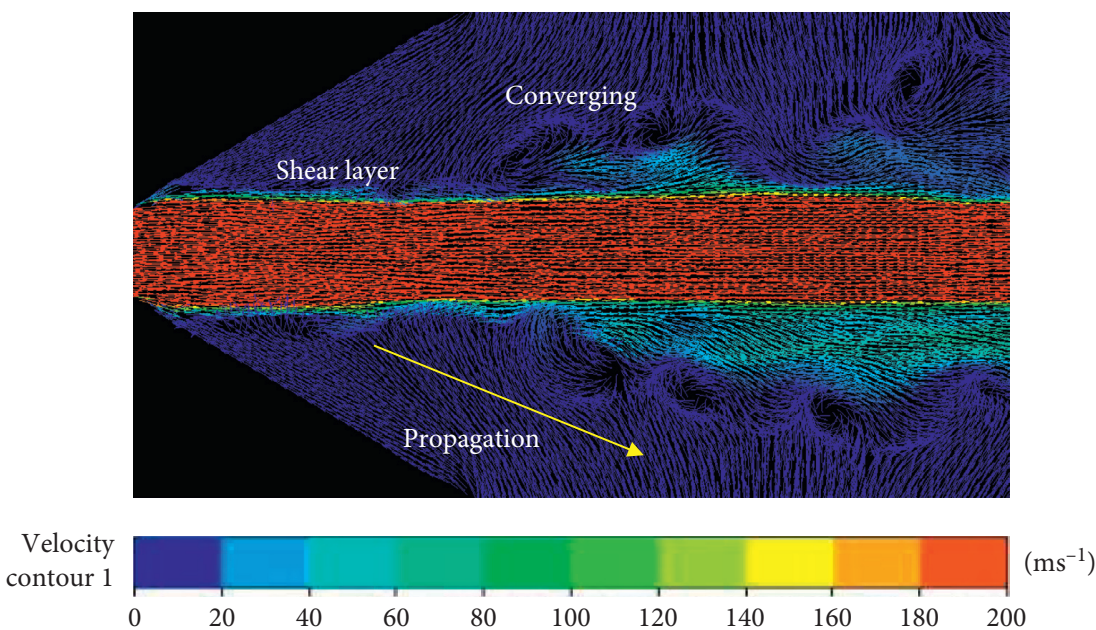

FIGURE 11: Vortex structure of the jet by a convergent-divergent nozzle.

the jet is changed, the pressure field, velocity field, and turbulence intensity in the jet will all change accordingly. In order to study the characteristics of the submerged cavitation jet under different pressure supplies, the aforementioned nozzle with an outlet angle of $40^{\circ}$ was used to calculate the jet flow field under different pressures. Figure 13 shows the isosurface with a vapor volume fraction of 0.2 . Through comparison, it can be seen intuitively that, with the increase of pressure, the distribution range of the cavitation cloud shows an expanding trend in both axial and radial directions, while the shape of the vacuole cloud is similar.
Figure 14 shows the distribution of vapor volume fraction inside and near the outlet of the nozzle under different cavitation numbers. The angle of the convergent section at the inlet of the angular nozzle throat is designed to be $13.5^{\circ}$ so that the fluid can enter the throat section smoothly. The transition structure effectively weakens the flow separation and vortex generation at the entrance of the throat, so there is almost no cavitation inside the nozzle. Cavitation starts to appear at the exit of the throat, which increases gradually with the decrease of the cavitation number. When $\sigma=0.0203$, cavitation mainly occurs at the 


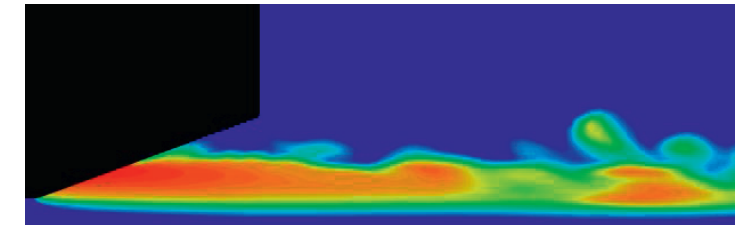

Phase 2 volume fraction

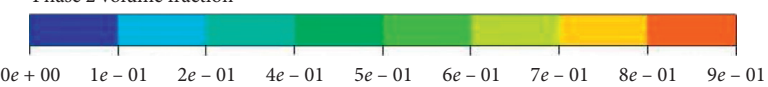

(a)

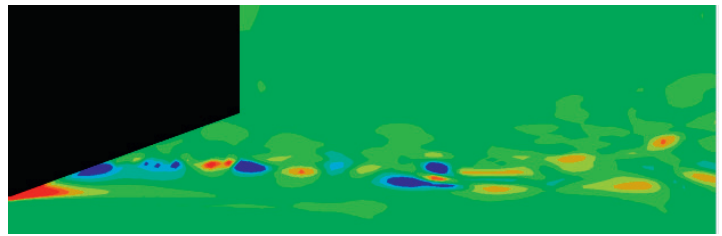

Velocity invariant Q $\left(\mathrm{s}^{-2}\right)$

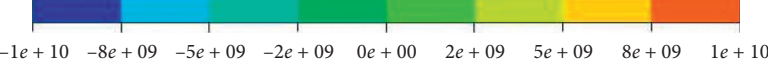

(c)

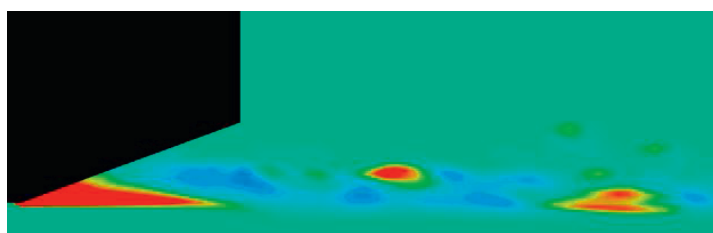

Mass transfer rate 1

$\left(\mathrm{kg} \mathrm{m}^{-3} \mathrm{~s}^{-1}\right)$

$-2 e+04-1 e+04-7 e+03 \quad 0 e+00 \quad 7 e+03 \quad 1 e+04 \quad 2 e+04 \quad 3 e+04 \quad 3 e+04 \quad 4 e+04$

(b)
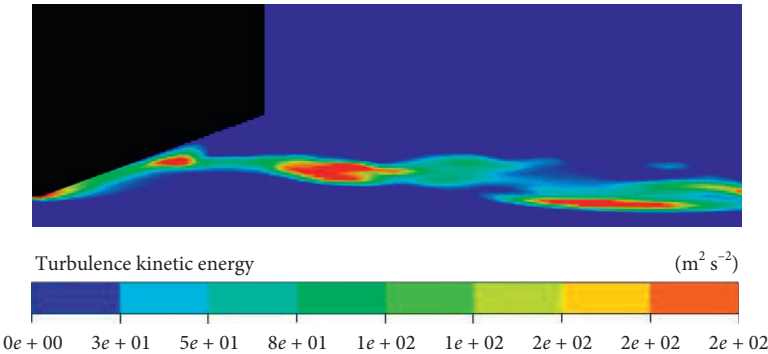

(d)

FIgURE 12: Distribution of variables at the angular nozzle outlet. (a) Vapor volume fraction. (b) Mass transfer rate. (c) Q-criteria. (d) Turbulence kinetic energy.

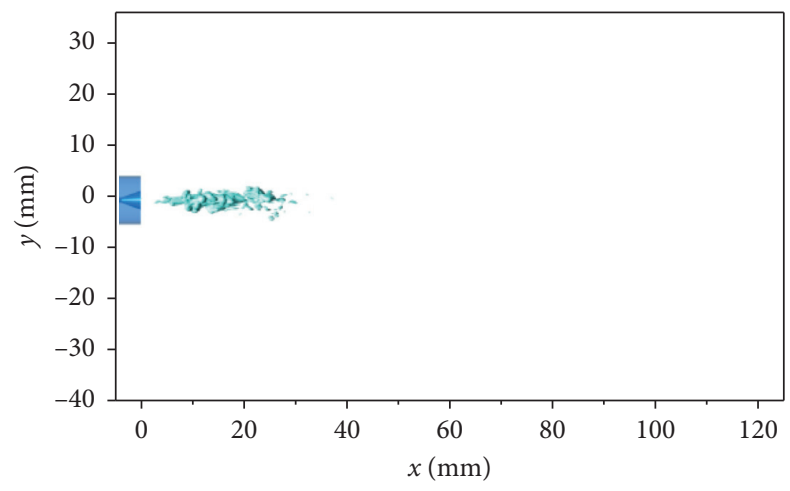

(a)

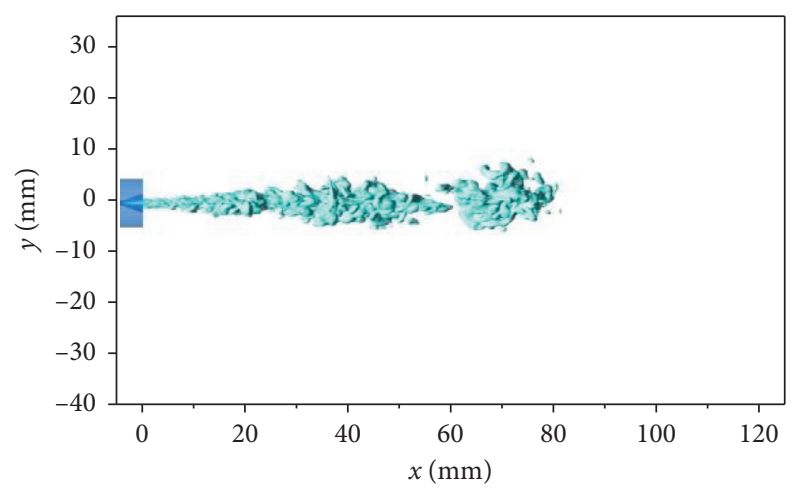

(c)

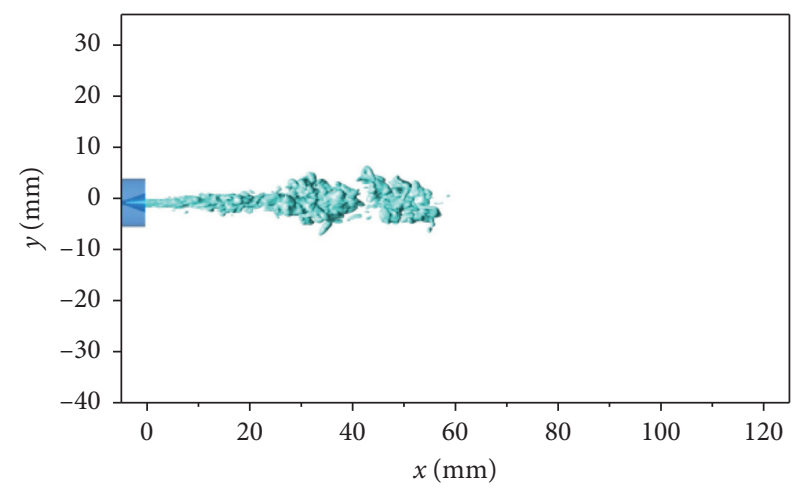

(b)

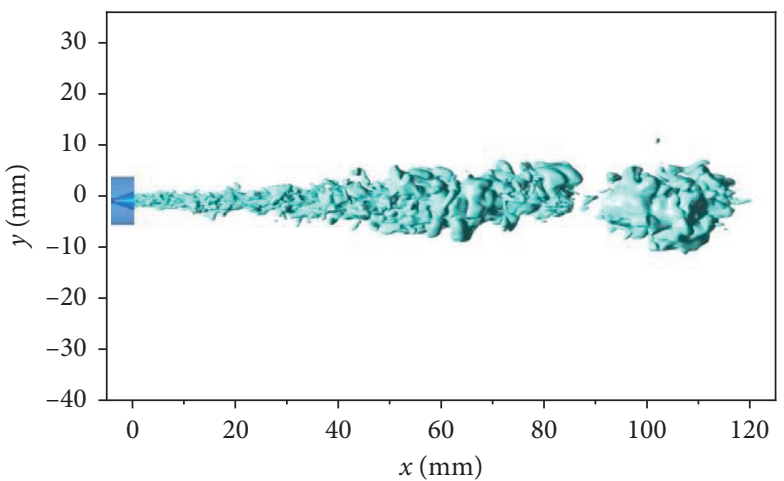

(d)

Figure 13: Isosurface of vapor volume fraction of the jet under different cavitation numbers. (a) $\sigma=0.0203$. (b) $\sigma=0.0101$. (c) $\sigma=0.0068$. (d) $\sigma=0.0051$. 


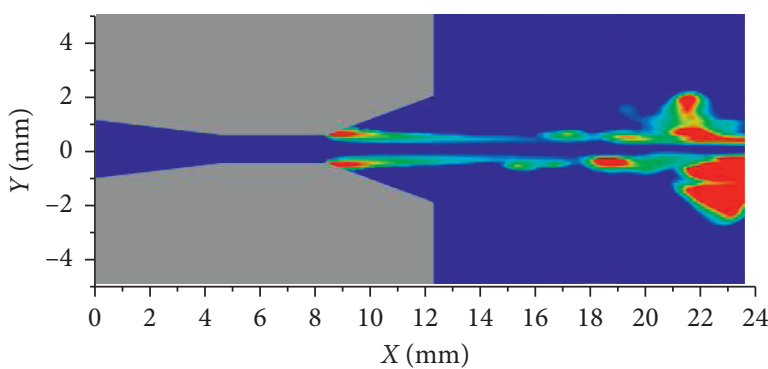

(a)

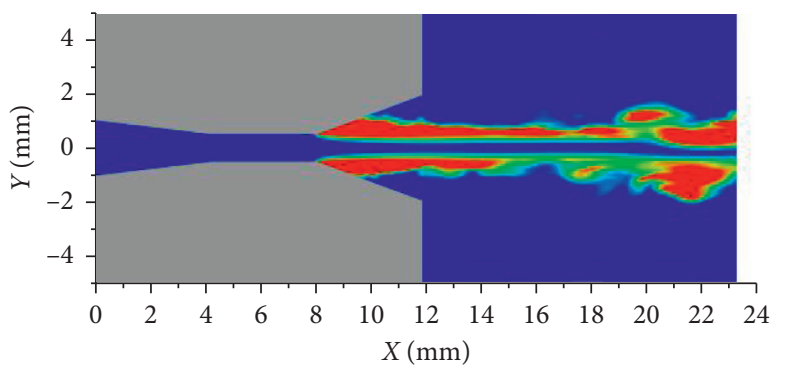

(c)

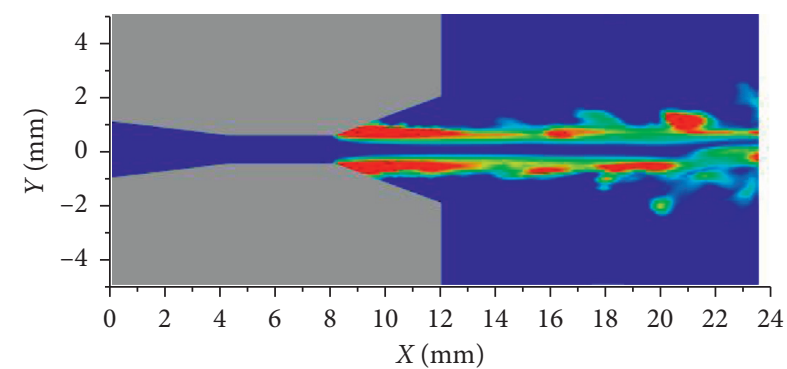

(b)

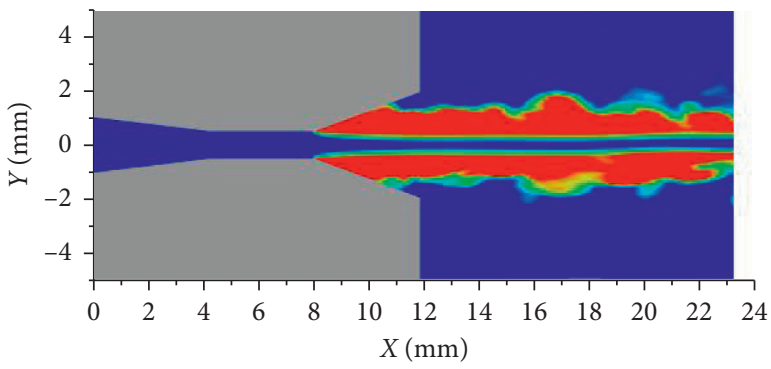

(d)

Figure 14: Vapor volume fraction under different cavitation numbers. (a) $\sigma=0.0203$. (b) $\sigma=0.0101$. (c) $\sigma=0.0068$. (d) $\sigma=0.0051$.

connection between the outlet of the throat and the whistle. At the outlet of the whistle, there is basically a low-concentration cavitation group. When $\sigma=0.0101$, the cavitation cloud extends to the whistle outlet, but the cavitation cloud in the external flow field is still intermittently distributed, shedding off from the outlet and moving downstream. When $\sigma=0.0068$, the length of the cavitation cloud generated in the whistle increases and extends to the downstream divergent section until it sheds off under the influence of the largescale vortex. When the cavitation number is further reduced to $\sigma=0.0051$, the cavitation is not limited to the inside of the nozzle because the shear layer with large viscous force is also expanded, and cavitation occurs within a certain distance from the nozzle outlet.

Figure 15 shows the jet velocity field under different cavitation numbers. The shape of the velocity field is similar to that of the vapor isosurface. Because the ambient pressure of the submerged jet remains unchanged, the turbulence intensity decreases with the decrease of the cavitation number. When the cavitation number is reduced from 0.0203 to 0.0051 , the range of the submerged jet increases from about $60 \mathrm{~mm}$ to more than $120 \mathrm{~mm}$. At high cavitation number conditions, the velocity core area in the nozzle exit section is relatively smooth, and the transition occurs downstream of the nozzle, while at low cavitation number, due to the high Reynolds number, the jet transitions inside the whistle. It accelerates the diffusion of the downstream flow field and makes the cavitation cloud more widely distributed.

Since the RANS-LES hybrid model has large fluctuations in the velocity and pressure in the high-turbulence area near the nozzle outlet, here, results of 400 time steps are timeaveraged during calculation to obtain the averaged velocity field. Figure 16 shows the radial distribution curve of jet velocity at different positions downstream of the nozzle under different cavitation numbers. Generally speaking, the jet velocity decays along the radial direction in all cases, which is similar to the curve shape in the experiment. Since the cavitation number here is adjusted by adjusting the pump pressure upstream of the nozzle, as the cavitation number decreases, the jet pressure gradually increases, and the nozzle outlet velocity also shows an increasing trend. When the cavitation number $\sigma=0.0203$, the maximum velocity at $10 \mathrm{~mm}$ downstream of the nozzle is $100 \mathrm{~m} / \mathrm{s}$. At this position, the jet has passed the turning section, and there is no obvious core area. When the cavitation number $\sigma=0.0101$, the maximum velocity at $10 \mathrm{~mm}$ downstream of the nozzle is $140 \mathrm{~m} / \mathrm{s}$, which is at the initial stage of the jet. The jet velocity first remains constant from the center along the radial direction and then begins to decrease. As the cavitation number continues to decrease, the characteristics of the core area at $x=10 \mathrm{~mm}$ become more obvious, and the maximum core velocity continues to increase.

The velocity under different cavitation numbers shown in Figure 16 is changed to a dimensionless form, where $u^{*}=u$ / $u_{0}$ and $y^{*}=y / y_{0.5}$. Figure 17 shows the radial distribution curve of the nondimensional axial velocity. It can be seen that the jet velocity distribution at different positions conforms to the characteristics of self-similarity, and the dimensionless velocity distribution curves under different cavitation numbers basically coincide, indicating that the cavitation number has little effect on the dimensionless distribution of jet velocity in the radial direction. According to the results of the PIV experimental data analysis, the radial distribution of the submerged cavitation jet velocity conforms to the exponential function. By Gaussian fitting, the dimensionless velocity distribution curve can be described as $u^{*}=e^{\left[-\left(r^{*} / 1.5\right)^{2}\right]}$. According to the comparison between the 


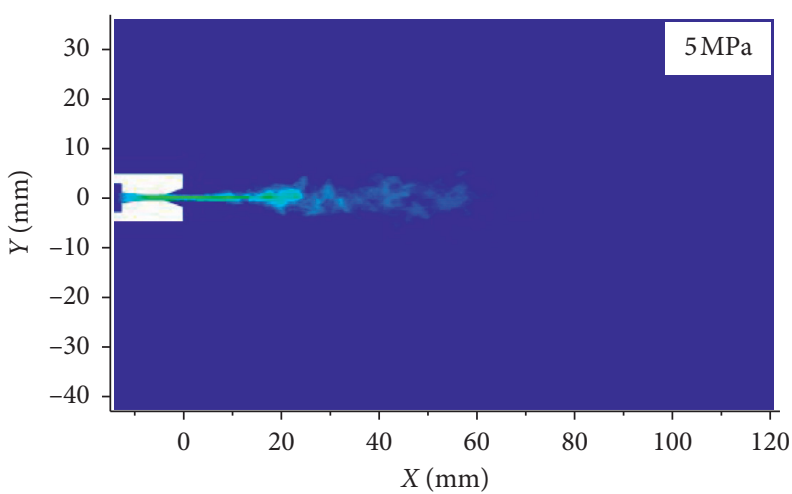

Velocity contour 1

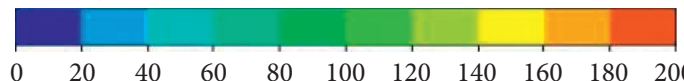

(a)

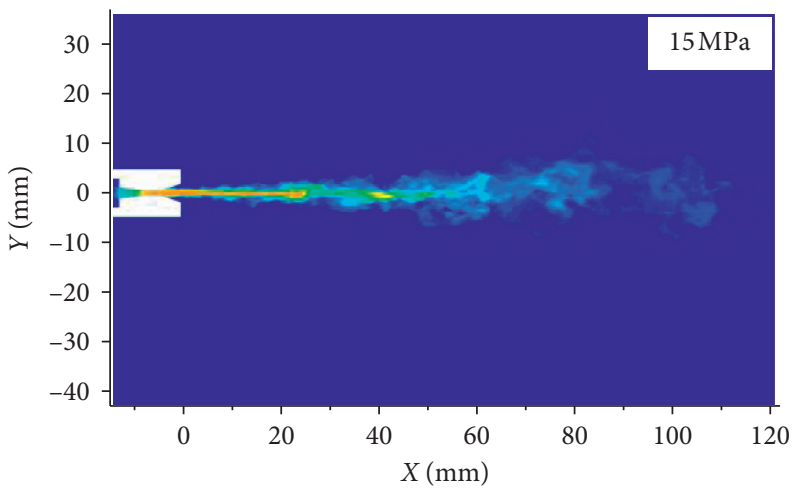

Velocity contour 1

$\left(\mathrm{ms}^{-1}\right)$

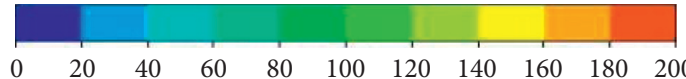

(c)

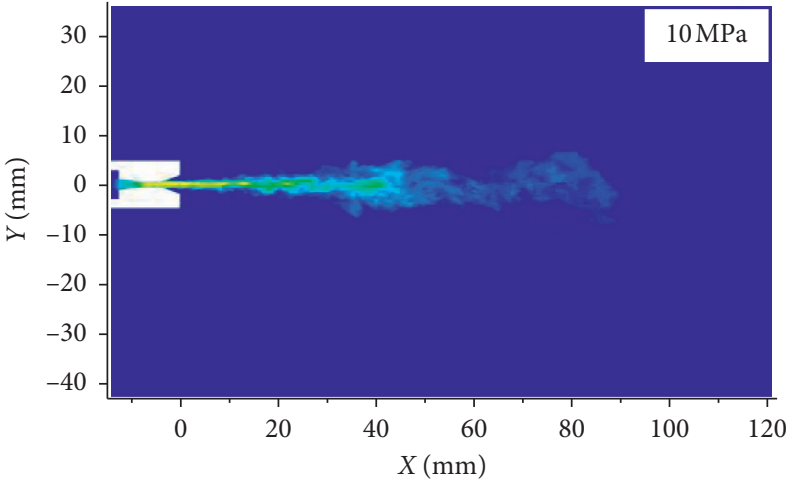

Velocity contour 1

$\left(\mathrm{ms}^{-1}\right)$

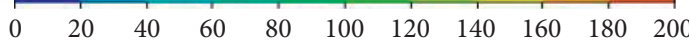

(b)

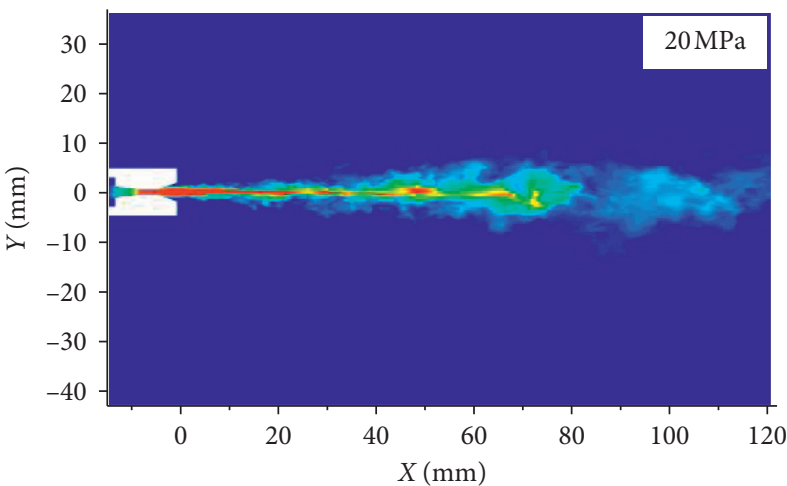

Velocity contour 1

$\left(\mathrm{ms}^{-1}\right)$

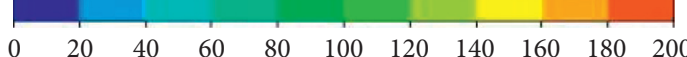

(d)

FIGURE 15: Velocity magnitude under different cavitation numbers. (a) $\sigma=0.0203$. (b) $\sigma=0.0101$. (c) $\sigma=0.0068$. (d) $\sigma=0.0051$.

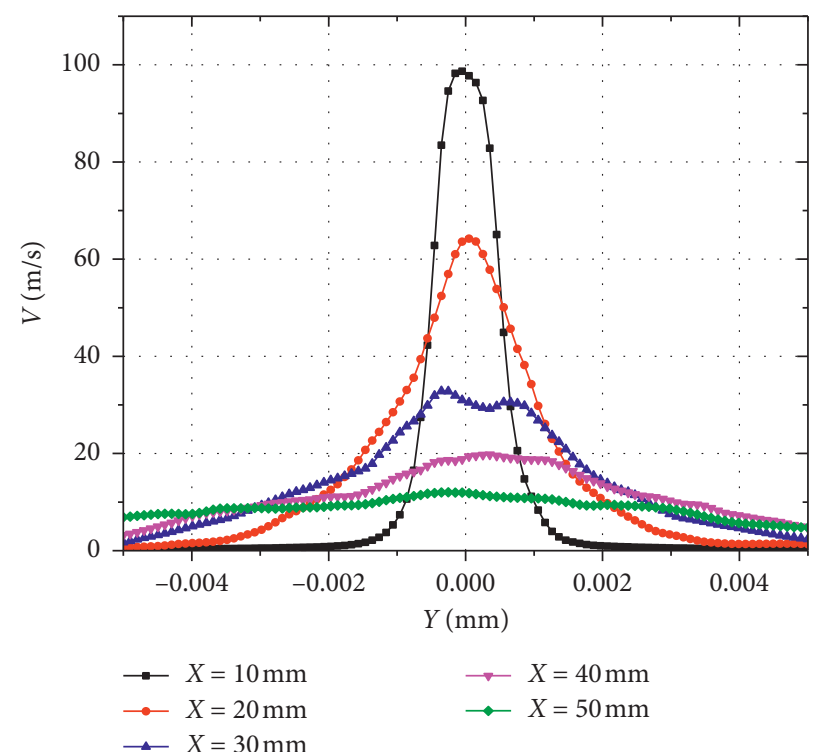

(a)

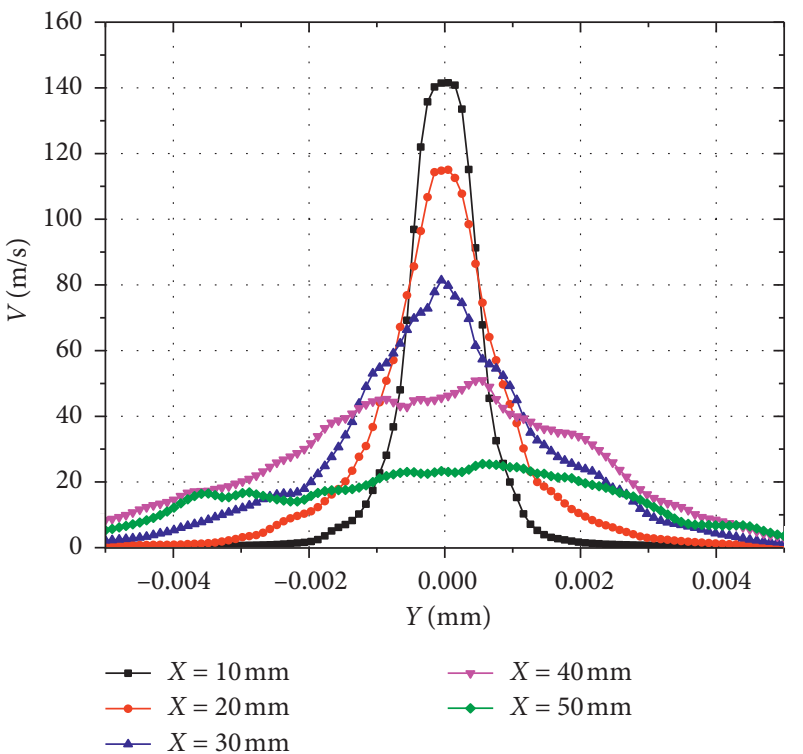

(b)

Figure 16: Continued. 


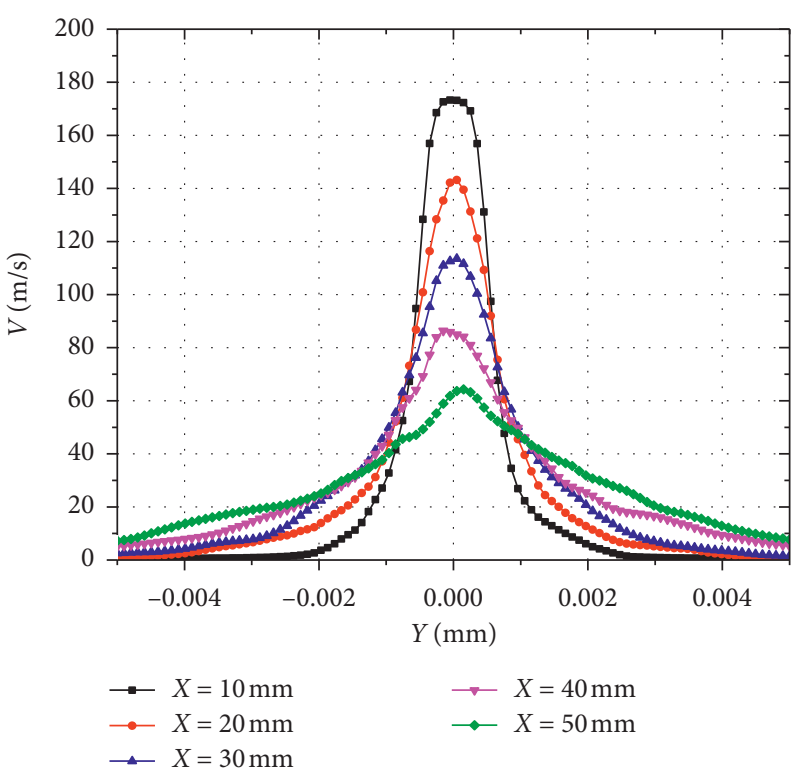

(c)

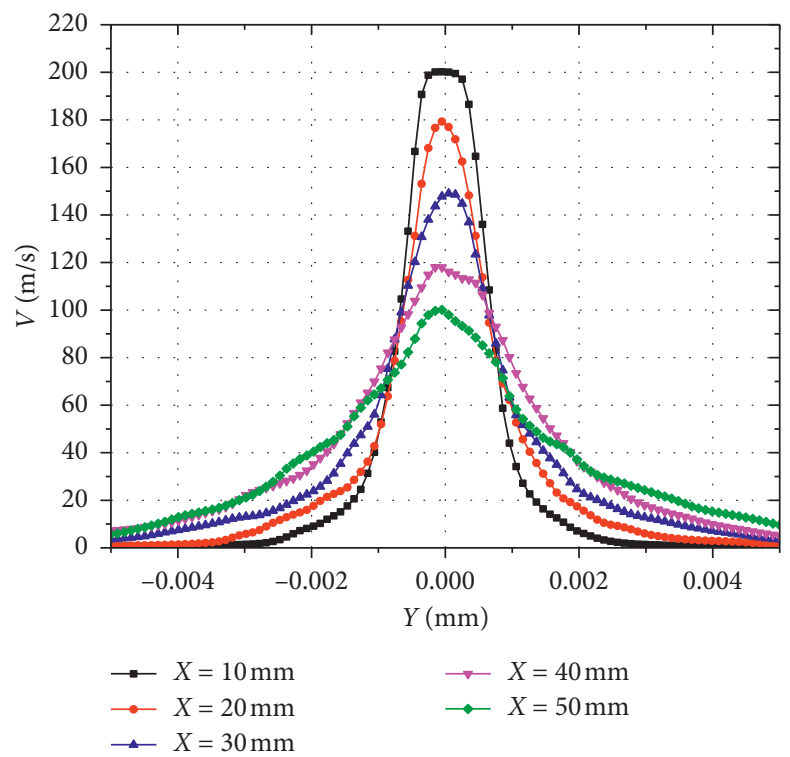

(d)

FiguRe 16: Velocity distributions along the radial direction under different cavitation numbers. (a) $\sigma=0.0203$. (b) $\sigma=0.0101$. (c) $\sigma=0.0068$. (d) $\sigma=0.0051$.

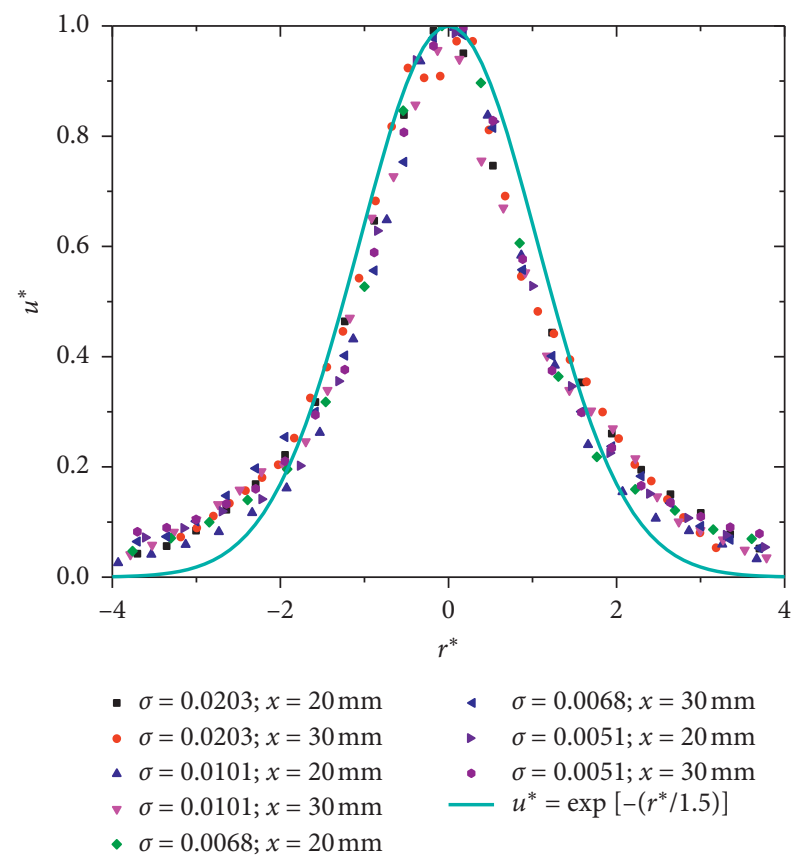

FIGURE 17: Normalized velocity along the radial direction under different cavitation numbers.

fitting function and the numerical calculation results in Figure 17, it can be seen that the formula is in good agreement with the calculation results, indicating that the fitting formula in the PIV experiment is applicable under different cavitation numbers.

\section{Conclusions}

In the current research, the submerged cavitation jet with different cavitation numbers is numerically investigated, and the effect of the turbulence model on the simulation results 
of the cavitation jet is analyzed. Three different turbulence models that represent the RANS and RANS-LES hybrid models are compared. The probability for the models to predict the vortex with small scale and the cavitation is evaluated in detail. The mechanism of the cavitation generation in the intensive shear layer of the submerged jet is revealed according to the simulation result, and the effect of the cavitation number on the characteristics of the submerged cavitation jet is analyzed as well. The following conclusions were obtained through research:

(1) The calculation probability for small-scale vortexes has a great influence on the prediction accuracy of cavitation in the submerged jet. The SST model is affected by the time-averaged process, which cannot predict the small-scale vortexes in the jet shear layer. Representative models of RANS-LES include the DES model and the SBES model. These two models can effectively capture the vortexes in the shear layer, and the SBES model can obtain more turbulence details than the DES model. The result of the simulation under different cavitation numbers using the SBES model agrees well with the experimental result.

(2) In the case of a low cavitation number, there exists an extensive shear layer at the exit of the corner nozzle, and small-scale vortexes will continue to form in the shear layer. According to the comparison of different physical parameters near the nozzle outlet, it is found that cavitation starts at the interface between the throat and the nozzle and mainly distributes along the boundary of the shear layer near the nozzle exit. The phase transition from liquid to cavitation is in a ring-shaped distribution, mainly located in the lowpressure area of the vortex center.

(3) According to the simulation results, it is found that, as the cavitation number decreases, the size and volume fraction of the cavitation bubbles increase significantly. The scale of the jet velocity field increases obviously with the decrease of the cavitation jet, while the profiles of the velocity distribution are similar to each other. The nondimensional velocity distribution along the radial direction shows a perfect similarity trend under each cavitation number, which follows a fitted exponential function.

\section{Data Availability}

The curved data used in this paper are available from the corresponding author upon request.

\section{Conflicts of Interest}

The authors declare that they have no conflicts of interest.

\section{Acknowledgments}

This research was funded by the National Key Research and Development Project of China (no. 2019YFB 2005300), National High-Tech Ship Scientific Research Project of
China (no. MIIT [2019] 360), National Natural Science Foundation of China (no. 51979138), Jiangsu Natural Science Research Project (no. 19KJB470029), Jiangsu Water Conservancy Science and Technology Project (no. 2019038), and Nantong Science and Technology Project (no. JC2019155).

\section{References}

[1] L. Sun, Y. J. Gong, Z. M. Zhang, and Z. W. Wang, "Software technology research on control system of ultra high pressure water jet rust removal equipment," Advanced Materials Research, vol. 721, pp. 372-376, 2013.

[2] B. Guo, T. A. G. Langrish, and D. F. Fletcher, "An assessment of turbulence models applied to the simulation of a two-dimensional submerged jet," Applied Mathematical Modelling, vol. 25, no. 8, pp. 635-653, 2001.

[3] L. Zhou, J. Liu, W. Chen, and D Chong, "Numerical investigation on steam jet submerged in subcooled water under different ambient pressures," International Communications in Heat and Mass Transfer, vol. 83, pp. 48-54, 2017.

[4] K. Manuela, G. Juergen, H. Alice, G. Christoph, and K. Christoph, "Numerical investigations of the extraction of submerged foundations by coupled CFD-DEM," in Proceedings of the ASME 2017 36th International Conference on Ocean, Offshore and Arctic Engineering, Trondheim, Norway, June 2017.

[5] S. Ragunathan, "Numerical simulation of the flow field of a confined, submerged slot jet impinging on an oscillating surface: a parametric study," in Proceedings of the ASME 2016 Fluids Engineering Division Summer Meeting Collocated with the ASME 2016 Heat Transfer Summer Conference and the ASME 2016 14th International Conference on Nanochannels, Microchannels, and Minichannels, American Society of Mechanical Engineers Digital Collection, Washington, DC, USA, July 2016.

[6] T. Xing, Z. Li, and S. H. Frankel, "Numerical simulation of vortex cavitation in a three-dimensional submerged transitional jet," Journal of Fluids Engineering, vol. 127, no. 4, pp. 714-725, 2005.

[7] X. Z. Song, Y. Zhang, F. Zhang et al., "Experimental investigation and CFD simulation of removal characteristics in nanoparticle colloid jet machining," Key Engineering Materials, vol. 431, pp. 273-276, 2010.

[8] C. Chen, S. Nie, Z. Wu et al., "A study of high pressure waterjet characteristics by CFD simulation," Machine Tool \& Hydraulics, vol. 2, pp. 103-105, 2006.

[9] S. G. Sagar, K. D. Sachin, B. J. Jyeshtharaj et al., "CFD simulation of flow pattern and plume dimensions in submerged condensation and reactive gas jets into a liquid bath," Chemical Engineering Science, vol. 63, no. 9, pp. 2420-2435, 2008.

[10] C. Wang, Q. Wu, B. Huang et al., "Numerical investigation of cavitation vortex dynamics in unsteady cavitating flow with shock wave propagation," Ocean Engineering, vol. 156, pp. 424-434, 2018.

[11] A. K. Singhal, M. M. Athavale, H. Li, and Y. Jiang, "Mathematical basis and validation of the full cavitation model," Journal of Fluids Engineering, vol. 124, no. 3, pp. 617-624, 2002.

[12] J. Sauer and G. H. Schnerr, "Unsteady cavitating flow-a new cavitation model based on a modified front capturing method and bubble dynamics," in Proceedings of the 2000 ASME Fluid 
Engineering Summer Conference, vol. 251, pp. 1073-1079, Boston, MA, USA, 2000.

[13] P. J. Zwart, A. G. Gerber, and T. Belamri, "A two-phase flow model for predicting cavitation dynamics," in Proceedings of the Fifth InternationalConference onMultiphase Flow, ICMF, Yokohama, Japan, June 2004.

[14] C. T. Hsiao, J. Ma, and G. L. Chahine, "Multiscale tow-phase flow modeling of sheet and cloud cavitation," International Journal of Multiphase Flow, vol. 90, pp. 102-117, 2017.

[15] H. Cheng, X. Long, B. Ji, X. Peng, and F. Mohamed, "A new Euler-Lagrangian cavitation model for tip-vortex cavitation with the effect of non-condensable gas," International Journal of Multiphase Flow, vol. 134, Article ID 103441, 2021.

[16] S. Xi, Z. Desheng, X Bin, S. B. Weidong, and B. P. M. van Esch, "Experimental and numerical investigation on the effect of tip leakage vortex induced cavitating flow on pressure fluctuation in an axial flow pump," Renewable Energy, vol. 163, pp. 1195-1209, 2021.

[17] Y. Yang, L. Zhou, J. Hang, D. Du, W. Shi, and Z. He, "Energy characteristics and optimal design of diffuser meridian in an electrical submersible pump," Renewable Energy, vol. 167, pp. 718-727, 2020.

[18] Y. Yang, L. Zhou, W. Shi, Z. He, Y. Han, and Y. Xiao, "Interstage difference of pressure pulsation in a three-stage electrical submersible pump," Journal of Petroleum Science and Engineering, vol. 196, Article ID 107653, 2021.

[19] M. Miltner, C. Jordan, and M. Harasek, "CFD simulation of straight and slightly swirling turbulent free jets using different RANS-turbulence models," Applied Thermal Engineering, vol. 89, pp. 1117-1126, 2015.

[20] A. J. Saddington, N. J. Lawson, and K. Knowles, "An experimental and numerical investigation of under-expanded turbulent jets," Aeronautical Journal, vol. 108, no. 1081, pp. 145-152, 2004.

[21] Z. Jaworski and B. Zakrzewska, "Modelling of the turbulent wall jet generated by a pitched blade turbine impeller," Chemical Engineering Research and Design, vol. 80, no. 8, pp. 846-854, 2002.

[22] P. Birkby and G. J. Page, "Numerical predictions of turbulent underexpanded sonic jets using a pressure-based methodology," Proceedings of the Institution of Mechanical Engineers-Part G: Journal of Aerospace Engineering, vol. 215, no. 3, pp. 165-173, 2001.

[23] Y. Wang, G. Yuan, Y. K. Yoon et al., "Large eddy simulation (LES) for synthetic jet thermal management," International Journal of Heat and Mass Transfer, vol. 49, no. 13-14, pp. 2173-2179, 2006.

[24] C. Wang, F. Wang, C. Ye, B. Wang, and Z. Zou, “Application of the MST turbulence model to predict the tip leakage vortex flows," Engineering Computations, vol. 38, no. 1, 2020.

[25] M. Manninen, V. Taivassalo, and S. Kallio, On the Mixture Model for Multiphase Flow, Valtion Teknillinen Tutkimuskeskus Publications, Finland, 1996.

[26] P. Zwart, A. G. Gerber, and T. Belamri, "A two-phase model for predicting cavitation dynamics," in Proceeding of the ICMF 2004 International Conference on Multiphase Flow, ICMF, Yokohama, Japan, May 2004.
[27] F. R. Menter, "Two-equation eddy-viscosity turbulence models for engineering applications," AIAA Journal, vol. 32, no. 8, pp. 1598-1605, 1994.

[28] P. R. Spalart, "Comments on the feasibility of LES for wings and on a hybrid RANS/LES approach," in Proceedings of the first AFOSR international conference on DNS/LES, Greyden Press, Ruston, Louisiana, August 1997.

[29] L. Yun, L. Xinping, J. Bin, H. Wenxin, and Q. Zhongdong, "Verification and validation of URANS simulations of the turbulent cavitating flow around the hydrofoil," Journal of Hydrodynamics, vol. 29, no. 4, pp. 610-620, 2017. 